12

\title{
Новый оптический метод исследования кислородной активности в текущей жидкости
}

\author{
() Р.В. Давыдов ${ }^{1}$, С.Э. Логунов ${ }^{1,2}$, В.И. Дудкин ${ }^{2}$, В.В. Давыдов ${ }^{1, \uparrow}$ \\ ${ }^{1}$ Санкт-Петербургский политехнический университет Петра Великого, \\ 195251 Санкт-Петербург, Россия \\ ${ }^{2}$ Санкт-Петербургский государственный университет телекоммуникаций им. проф. М.А. Бонч-Бруевича, \\ 195251 Санкт-Петербург, Россия \\ ฯ e-mail: Davydov_vadim66@mail.ru
}

Поступила в редакцию 24.09.2021 г.

В окончательной редакции 07.12.2021 г.

Принята к публикации 16.12.2021 г.

Обоснована необходимость исследования кислородной активности водных сред, находящихся в текущем состоянии для решения различных задач. Установлено, что испускаемые $\gamma$-кванты в результате распада ядер ${ }^{16} \mathrm{~N}$ с энергией более $9 \mathrm{MeV}$ вызывают образование дополнительных центров окраски и обратимых оптических дефектов в волокне. Их появление приводит к увеличению радиационно-наведенных потерь в оптическом волокне, которые уменьшают мощность лазерного излучения, передаваемого по нему. Разработан новый оптический метод, позволяющий по изменению числа $\gamma$-квантов, испущенных ядрами ${ }^{16} \mathrm{~N}$ при движении жидкости по трубопроводу, исследовать характер изменения кислородной активности. Впервые исследован характер изменения спектрального распределения количества образовавшихся ядер ${ }^{16} \mathrm{~N}$ в результате кислородной активности и определены ее особенности в текущем потоке. Для реализации долговременных по времени исследований кислородной активности предложен способ восстановления оптических свойств волокна в присутствии фонового радиоактивного излучения.

Ключевые слова:текущая жидкость, ядра $16 \mathrm{~N}$, гамма-кванты, кислородная активность теплоносителя.

DOI: $10.21883 /$ OS.2022.03.52173.2765-21

\section{Введение}

Развитие атомной энергетики, необходимость в проведении исследований для отработки методик по управлению цепными реакциями в ядерных реакторах нового поколения поставили перед учеными ряд сложных задач [1-4], одна из которых - это исследование характера изменения кислородной активности жидких сред, применяемых в качестве теплоносителя в ядерных реакторах, при движении их по трубопроводу.

В настоящее время на АЭС контроль состояния кислородной активности в потоке теплоносителя не реализован $[1,4]$. Контролируется содержание ${ }^{131} \mathrm{I}$, так как это характеризует отравление зоны реактора ксеноном. В ряде случаев контролируют активность продуктов коррозии. В новых моделях реакторов осуществляется контроль реперных нуклидов, в первую очередь ${ }^{60} \mathrm{Co}$, так как это характеризует герметичность топлива. В последних моделях реакторов на АЭС реализован непрерывный контроль за величиной протечки теплоносителя из первого контура во второй на основе регистрации $\gamma$-излучения изотопа ${ }^{16} \mathrm{~N}$ в паре. Это позволяет предотвратить аварию на АЭС в зоне парогенератора [5]. Опыт эксплуатации данной системы показал, что для исследования кислородной активности теплоносителя в потоке (особенно продолжительное время) данный метод не приемлем.

Данные о характере изменения кислородной активности необходимы для определения состояния (или контроля состояния) теплоносителя, что необходимо для обеспечения безопасной работы реактора. Кроме того, специалисты в области ядерной энергетики предполагают, что данные об изменении кислородной активности теплоносителя во времени позволят получить новую информацию о характере протекания цепных реакций $[1,6]$. Это необходимо для разработки новых моделей реакторов.

Поэтому целью настоящей работы является разработка нового метода исследования кислородной активности в потоке теплоносителя.

\section{Метод исследования кислородной активности в потоке теплоносителя}

Кислородная активность ${ }^{16} \mathrm{O}(n, p){ }^{16} \mathrm{~N}$ связана с взаимодействием ядер кислорода, входящими в состав одной из частей теплоносителя - воды, с нейтронами с энергией более $9 \mathrm{MeV}$, которые присутствуют в зоне реактора в большом количестве по причине протекания цепной реакции. В результате этого взаимодействия образуется ядро ${ }^{16} \mathrm{~N}$, которое крайне нестабильно. После образования ядра начинается процесс его распада. Период полураспада составляет порядка $7.1 \mathrm{~s}$. Распад ${ }^{16} \mathrm{~N}$ сопровождается $\gamma$-излучением с энергией $E_{\gamma} \approx 6.2 \mathrm{MeV}$.

Для исследования данного явления в научных лабораториях используются специально разработанные 


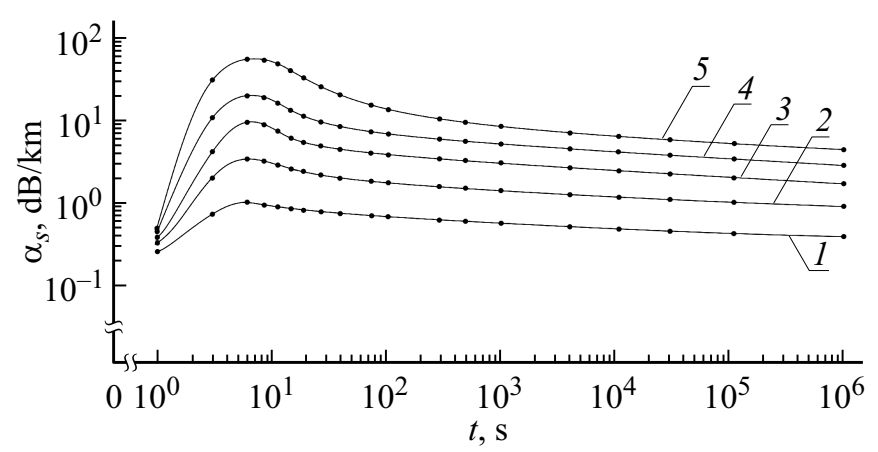

Рис. 1. Изменение оптических потерь $\alpha_{s}$ от времени $t$ в одномодовом волокне при $T=294.2 \mathrm{~K}$. Длина волны лазерного излучения $\lambda=1550 \mathrm{~nm}$. Графикам $1,2,3,4$ и 5 соответствуют различные концентрации оксида германия в сердцевине оптического волокна $\mathrm{SiO}_{2}-\mathrm{GeO}_{2}$ (в \%): 0, 1.5, 4, 10, 20 .

ионизационные камеры, но их габариты и особенности конструкций исключают возможность применения в реакторном отделении. Также крайне сложно использовать в реакторном отделении для исследования кислородной активности ряд промышленных приборов, например „АZOT-16-PG“, предназначенных для определения утечки теплоносителя между первым и вторым контуром по регистрации $\gamma$-квантов в паре.

Поэтому нами был разработан следующий метод. Проведенные ранее исследования показали, что под действием $\gamma$-излучения оптическое волокно темнеет (возрастают радиационно-наведенные потери, $\alpha_{s}$ ) [7-9]. Для измерения $\alpha_{s}$ используется классическая формула

$$
\alpha_{s}=-10 \lg \left(P_{\text {out }} / P_{\text {in }}\right) / l,
$$

где $P_{\text {in }}-$ мощность лазерного излучения, вводимая в оптическое волокно, $P_{\text {out }}-$ мощность на выходе из оптического волокна, $l$ - длина оптического волокна.

Эффект возрастания $\alpha_{s}$ основан на образовании электронно-дырочных пар. Выбивая кислород, электрон занимает его место в циклической пространственной структуре $[7,8,10]$. Образуются различные центры окраски и „электронные“ соединения с различными временами жизни [7-12].

Проведенные исследования [7-13] показали, что если экспозиционная доза облучения невысокая, то число образовавшихся центров окраски и ,электронных“ соединений невелико. Деструкция в сетке стекла не происходит. Прозрачность оптических волокон сохраняется достаточно долго. Волокно темнеет слабо. С увеличением экспозиционной дозы облучения число этих образований увеличивается, возрастает скорость потемнения волокна. Возрастают потери $\alpha_{s}$. На рис. 1 представлены результаты исследований изменения $\alpha_{s}$ от времени $t$ при кратковременном воздействии на него $\gamma$-излучения, длительность которого порядка 6-7s (экспозиционная доза облучения оптического волокна составляет $100 \mathrm{~Gy}$ ), для различного состава сердцевины оптического волокна.
Эти исследования проводились на экспериментальном стенде, конструкция которого и принцип проведения измерений схожи с экспериментальной установкой и измерениями, представленными в [13].

Полученные результаты показывают, что, изменяя легирование сердцевины оптического волокна (концентрацию в ней оксида германия $\mathrm{GeO}_{2}$ ), можно изменить чувствительность волокна к воздействию $\gamma$-излучения и установить рабочую точку при регистрации $\gamma$-излучения на участке зависимости $\alpha_{s}(t)$ с максимальной крутизной склона (рис. 1). Это позволит в ряде случаев регистрировать всплески кислородной активности от распада небольшого числа ядер ${ }^{16} \mathrm{~N}$.

После прекращения действия $\gamma$-излучения оптические свойства волокна со временем восстанавливаются. Исследования показали, что процессы восстановления прозрачности в оптическом волокне после воздействия $\gamma$ излучения протекают крайне медленно (процесс может длится более $10^{7} \mathrm{~s}$ ) [8-15]. В ряде случаев (при больших экспозиционных дозах облучения стекла [8]) процесс восстановления оптических свойств не происходит в течении 38 лет. Это означает, что при образовании большого числа центров окраски также произошла деструкция (деформация) сетки стекла. Релаксация центров окраски замедлилась скорее всего по причине деструкции (стекло не светлеет). Излучение большой мощности (в ряде случаев более $2.5 \mathrm{~W}$ ) на различных длинах волн через стекло не проходит.

В отличие от многочисленных экспериментальных исследований, связанных с воздействием $\gamma$-излучения на оптическое волокно, в специальной зоне реакторного отделения, где расположены катушки с оптическим волокном, мощность дозы соизмерима с тем, что воздействует на волокно, расположенное на спутнике на орбите [8]. Деструкция сетки стекла при таких экспозиционных дозах облучения в волокне происходить не будет. Поэтому можно для очистки волокна от центров окраски и ,электронных“ соединений использовать разработанный метод с использованием дополнительного лазерного излучения, который также применяется другими учеными $[13,16,17]$ при исследованиях.

На рис. 2 в качестве примера представлен процесс управления релаксационными процессами центров окраски и „электронных“ соединений в оптическом волокне после прекращения импульсного воздействия $\gamma$-излучения с экспозиционной дозой облучения $100 \mathrm{~Gy}$ при сохранении радиоактивного фона.

Полученные результаты позволили установить, что с увеличением легирования сердцевины оптического волокна оксидом германия скорость очистки оптического волокна под действием вынужденного лазерного излучения возрастает.

При протекании по трубопроводу теплоносителя с кислородной активностью происходит испускание дополнительных $\gamma$-квантов к „естественному“ фону радиоактивного излучения. Проведенные ранее исследования на экспериментальной модели реактора Брест- 


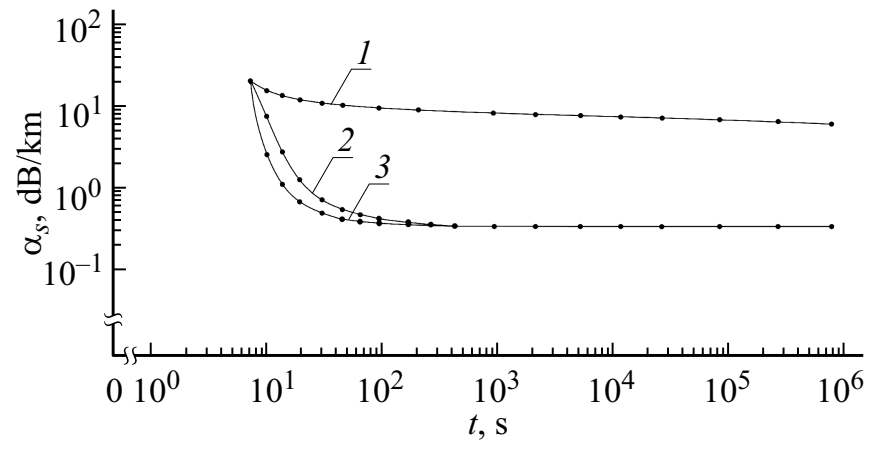

Рис. 2. Изменение оптических потерь $\alpha_{s}$ от времени $t$ в одномодовом волокне с сердцевиной $\mathrm{SiO}_{2}-\mathrm{GeO}_{2}$ (легирование $10.0 \%$ ) при $T=294.3 \mathrm{~K}$. Длина волны лазерного излучения $\lambda=1550 \mathrm{~nm}$. Графикам 1,2 и 3 соответствует мощность импульсного лазерного излучения на длине волны $\lambda=547 \mathrm{~nm}$ (в $\mathrm{mW}): 0,140,380$.

300 показали, что данная добавка к экспозиционной дозе облучения, действующая на оптическое волокно, носит импульсный характер и появляется в определенной зоне трубопровода по причине неравномерного распределения в теплоносителе нейтронов с энергией более $9 \mathrm{MeV}$, которые при взаимодействии с ядрами кислорода, входящими в состав теплоносителя, образуют ядра ${ }^{16} \mathrm{~N}$ в результате реакции $(n, p)$. Исследования кислородной активности в реакторе Брест-300 показали, что максимальный временной интервал между появлениями этих всплесков $\gamma$-излучения в фиксированной зоне трубопровода составляет менее $250 \mathrm{~s}$.

Поэтому разработанный процесс управления релаксационными процессами в оптическом волокне позволит проводить продолжительные исследования кислородной активности теплоносителя в трубопроводе. Без использования быстрой „очистки“ оптическое волокно будет темнеть. Чувствительность оптического волокна к новым воздействиям $\gamma$-излучения будет снижаться. Использование дополнительного лазерного излучения позволяет вернуть рабочую точку после регистрации $\gamma$ излучения от кислородной активности на участок зависимости $\alpha_{s}(t)$ с максимальной крутизной склона (рис. 1). Это обеспечивает воспроизводимость чувствительности при повторных измерениях.

Кроме того, ранее проведенные нами исследования влияния $\gamma$-излучения на образования радиационнонаведенных потерь в оптическом волокне [15-17] позволили установить, что при определенной мощности лазерного излучения $P_{\text {in }}$ может установиться равновесие между двумя процессами (образованием новых и релаксацией существующих центров окраски и „электронных“ соединений) при отсутствии других факторов, которые при воздействии $\gamma$-излучения существенно влияют на $\alpha_{s}(t)$. В этом случае регистрируемая мощность лазерного излучения на выходе оптического волокна $P_{\text {out }}$ остается неизменной. Это означает, что выполняется следующее соотношение:

$$
P_{\text {in }}=P_{\text {out }}+P_{\text {rel }},
$$

где $P_{\text {rel }}-$ мощность лазерного излучения, израсходованная на релаксацию определенного количества $(N)$ центров окраски и „электронных“ соединений, которые приводят к затуханию оптического сигнала в волокне.

Введем мощность $\gamma$-квантов $P_{\gamma}$, которая была затрачена на образование $N$ новых центров окраски и „электронных“ соединений в оптическом волокне. Эту мощность можно определить следующей формулой:

$$
P_{\gamma}=E / t_{\gamma},
$$

где $E$ - энергия на образования $N$ новых центров окраски и „электронных“ соединений, $t_{\gamma}$ - время воздействия $\gamma$-квантов на оптическое волокно.

Будем считать, что при распаде ядра ${ }^{16} \mathrm{~N} \gamma$-кванты излучаются во все направления одинаково. В этом случае они равномерно взаимодействуют с оптическим волокном, которое намотано в один слой на теплоизоляцию трубопровода, по которому течет теплоноситель. Согласно работам $[7,10,12,13]$ и квантовой теории поля $[18,19]$, в первом приближении при взаимодействии одного $\gamma$-кванта с оптическим волокном образуется один центр окраски или одно „электронное“ соединение. В этом случае значение $E$ можно вычислить с использованием следующего соотношения:

$$
E=S N h v / \sigma=S N h c /(\lambda \sigma),
$$

где $\sigma$ - сечение взаимодействия (рассеяния) $\gamma$-кванта с атомами кислорода $\mathrm{O}_{2}$ в оптическом волокне, $S-$ площадь взаимодействия оптического волокна с $\gamma$-квантами, $N$ - число испущенных $\gamma$-квантов, $\lambda-$ длина волны излучения $\gamma$-кванта.

Площадь взаимодействия $S$ можно определить по следующей формуле:

$$
S=\pi D_{p} n_{f} d_{f} / 2
$$

где $D_{p}$ - диаметр трубопровода с теплоизоляцией, $n_{f}$ - число витков оптического волокна, $d_{f}$ - диаметр оптического волокна.

При распаде ядра ${ }^{16} \mathrm{~N}$ испускание $\gamma$-квантов соответствует 7F-линии спектра $(\lambda=0.254 \mathrm{~nm})$. Тогда значение $N$ можно определить, используя следующее соотношение:

$$
N=\sigma \lambda t_{\gamma}\left(P_{\text {in }}-P_{\text {out }}\right) /(S h c) .
$$

В соотношении (6) значения мощностей лазерного излучения $P_{\text {in }}$ и $P_{\text {out }}$ измеряются. Значение $\sigma$ устанавливается экспериментально для каждого типа волокна, так как оно будет зависеть от легирования сердцевины оптического волокна оксидом германия $\mathrm{GeO}_{2}$, а также от температуры оптического волокна $T_{f}$. Например, для оптического волокна, легированного $1.5 \%$ оксидом германия $\mathrm{GeO}_{2}$, при $T=295.3 \mathrm{~K} \sigma \approx 4.7 \cdot 10^{-15} \mathrm{~cm}^{2}$. Это 


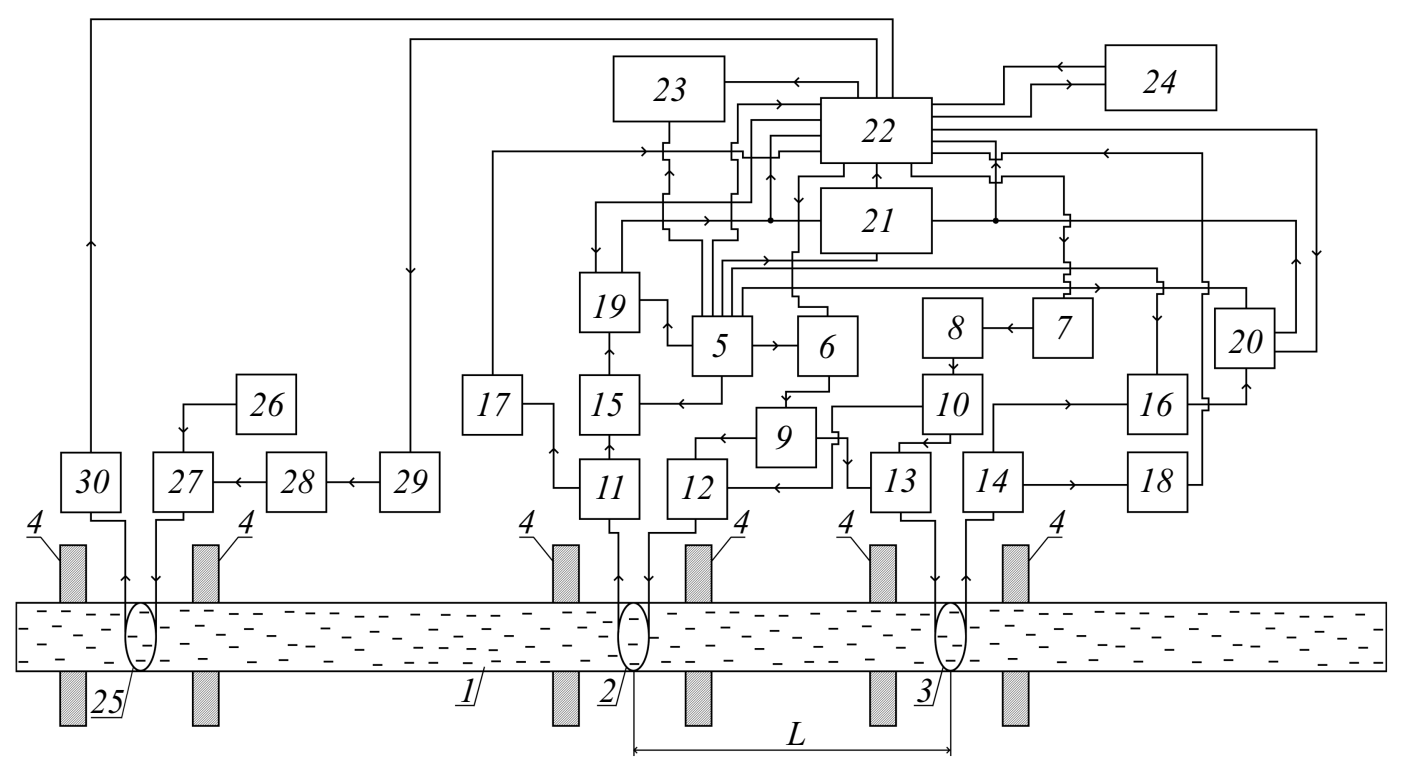

Рис. 3. Структурная схема волоконно-оптической системы: 1 - трубопровод с теплоносителем; 2,3 и 25 - катушки с оптическим волокном (датчики); 4 - защитные радиационные экраны; 5 - многофункциональный драйвер питания; 6 и 26 - передающие оптические модули; 7 и 29 - многофункциональные драйверы питания; 8 и $28-$ полупроводниковые лазеры; 9 и $10-$ оптические делители (1/2); 11-14 и 27 - мультиплексоры; 15, 16 и $30-$ фотоприемники; 17 и 18 - измерители оптической мощности; 19 и 20 - компараторы; 21 - логическое устройство; 22 - устройство обработки и управления; 23 - устройство индикации; 24 - центральный компьютер.

значение хорошо согласуется с данными, полученными другими учеными [20-22]. Это подтверждает адекватность используемой нами методики измерения $\sigma$ для определения $N$ с использованием формулы (6). Кроме контроля значений температур (теплоносителя, $T_{c}$, и $T_{f}$ ) при проведении исследований кислородной активности необходимо контролировать расход теплоносителя $q$, так как значение $t_{\gamma}$ определяется из следующего соотношения: $t_{\gamma}=\pi L_{\mathrm{ef}} d_{p}^{2} / 4 q\left(L_{\mathrm{ef}}-\right.$ эффективная длина трубопровода, $d_{p}-$ его диаметр). Поэтому функцию измерения $q$ также необходимо реализовать в разрабатываемой нами волоконно-оптической системе.

\section{Волоконно-оптическая система для исследования кислородной активности в потоке теплоносителя}

Для исследования параметров кислородной активности нами была разработана волоконно-оптическая система. Ее структурная схема представлена на рис. 3 . В конструкции системы полностью исключен контакт измерительных элементов с теплоносителем. Катушки 2, 3 и 25 с оптическим волокном размещены поверх теплоизоляции трубопровода 1. Данная схема размещения катушек 2,3 и 25 предполагает два возможных варианта исследования кислородной активности. Это неподвижные каркасы (расстояние $L$ фиксировано, см. рис. 3) или подвижные каркасы - расстояние $L$ изменяется. В этом случае также может измениться расстояние между катушками $L_{1}$ (рис. 3). Последнее расширяет функциональные возможности разработанной нами системы.

Необходимо отметить, что в условиях эксплуатации АЭС расстояния $L$ изменять крайне сложно и не всегда целесообразно. Функция изменения $L$ и $L_{1}$ необходима в случае проведения различных исследований на экспериментальной модели реактора, так как значение расхода теплоносителя $q$ при проведении ряда экспериментов необходимо изменять в большом диапазоне (минимум на два порядка).

Отметим основные моменты работы разработанной нами волоконно-оптической системы. От передающего оптического модуля 6 излучение с $\lambda=1550 \mathrm{~nm}$ (мощность излучения $P_{m}$ регулируется от 0.1 до $20 \mathrm{~mW}$ ) через оптический делитель $9(N=2)$ поступает на входы мультиплексоров 12 и 13 . Длина волны $\lambda=1550 \mathrm{~nm}$ для измерения потерь $\alpha_{s}(t)$ лазерного излучения выбрана по следующим причинам. С увеличением $\lambda$ уменьшается коэффициент релеевского рассеяния (КРР). Кроме того, „хвосты“ фононного и электронного поглощения в области длин волн $1.0-1.6 \mu \mathrm{m}$ не дают существенного вклада в оптические потери, данные о которых используются для исследования изменения характера кислородной активности теплоносителя.

На другие входы этих мультиплексоров подается лазерное излучение с $\lambda=457 \mathrm{~nm}$ от полупроводникового лазера 8 с диодной накачкой (SSP-ST-457-F). Мощность излучения $P_{L}$ регулируется от 1 до $1000 \mathrm{~mW}$. Многофункциональный драйвер питания 7 позволяет 
работать лазеру 8 как в непрерывном, так и в импульсном режиме. Длина волны из диапазона лазерного излучения 440-485 nm (синей части спектра) для управления скоростью релаксации центров окраски и „электронных“ соединений выбрана по следующим причинам. С одной стороны, при больших длинах оптического волокна необходимо обеспечить наименьшее значение КРР, чтобы дополнительное лазерное излучение провело максимальную очистку стекла. С другой стороны, нижняя граница лазерного излучения при температуре $T=307 \mathrm{~K}$ для эффективной очистки $E^{\prime}$-центров составляет $\lambda=217 \mathrm{~nm}$ [11]. Все остальные центры окраски, которые изменяют оптические свойства лазерного излучения, проходящего через волокно, имеют области потерь в более длинноволновой части спектра [7]. При очистке волокна желательно уменьшить потери всех видов. С учетом того, что у нас температура намного больше, чем в [11], было выбрано лазерное излучение с $\lambda=457 \mathrm{~nm}$, так как есть вероятность, что оно может задеть „хвост “ спектра $E^{\prime}$-центров, так как при больших температурах он смещается и расширяется.

В зонах размещения катушек 2,3 и 26 температура для базовых режимов работы АЭС изменяется от 343 до $593 \mathrm{~K}$ в зависимости от модели реактора. В случае аварийных режимов работы температура может увеличиться до $1300 \mathrm{~K}$ и более. Это приведет к разрушению разработанной системы, так как в ней используется устойчивое к высоким температурам (до $980 \mathrm{~K}$ ) одномодовое волокно (компания Fiberguide, USA) с медноникелевым покрытием.

Также необходимо учитывать, что длина оптического волокна $l$ может быть $1000 \mathrm{~m}$ и более. Поэтому желательно сместиться в сторону увеличения $\lambda$ для уменьшения КРР. Стоит отметить, что в выпускаемых промышленных лазерах на $\lambda=457 \mathrm{~nm}$ можно, используя многофункциональный драйвер питания, обеспечить перестройку мощности лазерного излучения с единиц $\mathrm{mW}$ до $1 \mathrm{~W}$ в непрерывном режиме, а в импульсном значительно больше.

Выходы мультиплексоров 12 и 13 подключены к катушкам оптического волокна 2 и 3. С катушек оптического волокна 2 и 3 лазерное излучение двух длин волн поступает на выходы мультиплексоров 11 и 14 (входы этих мультиплексоров подключены к входам измерителей мощности 17 и 18 и фотоприемников 15 и 16). На измерители мощности 17 и 18 поступает лазерное излучение с $\lambda=457 \mathrm{~nm}$, на фотоприемники 15 и 16 - лазерное излучение с $\lambda=1550 \mathrm{~nm}$. Измерители мощности необходимы для контроля состояния оптического волокна (определения коэффициента радиационнонаведенных потерь $\alpha_{s}$ ) с использованием (1). В разработанной нами конструкции волоконно-оптической системе $l$ - это расстояние от выхода мультиплексора 12 или 13 до выхода мультиплексора 11 или 14. Кроме того, в этой системе реализована возможность измерения значения $q$. Для этого с фотоприемников 15 и 16
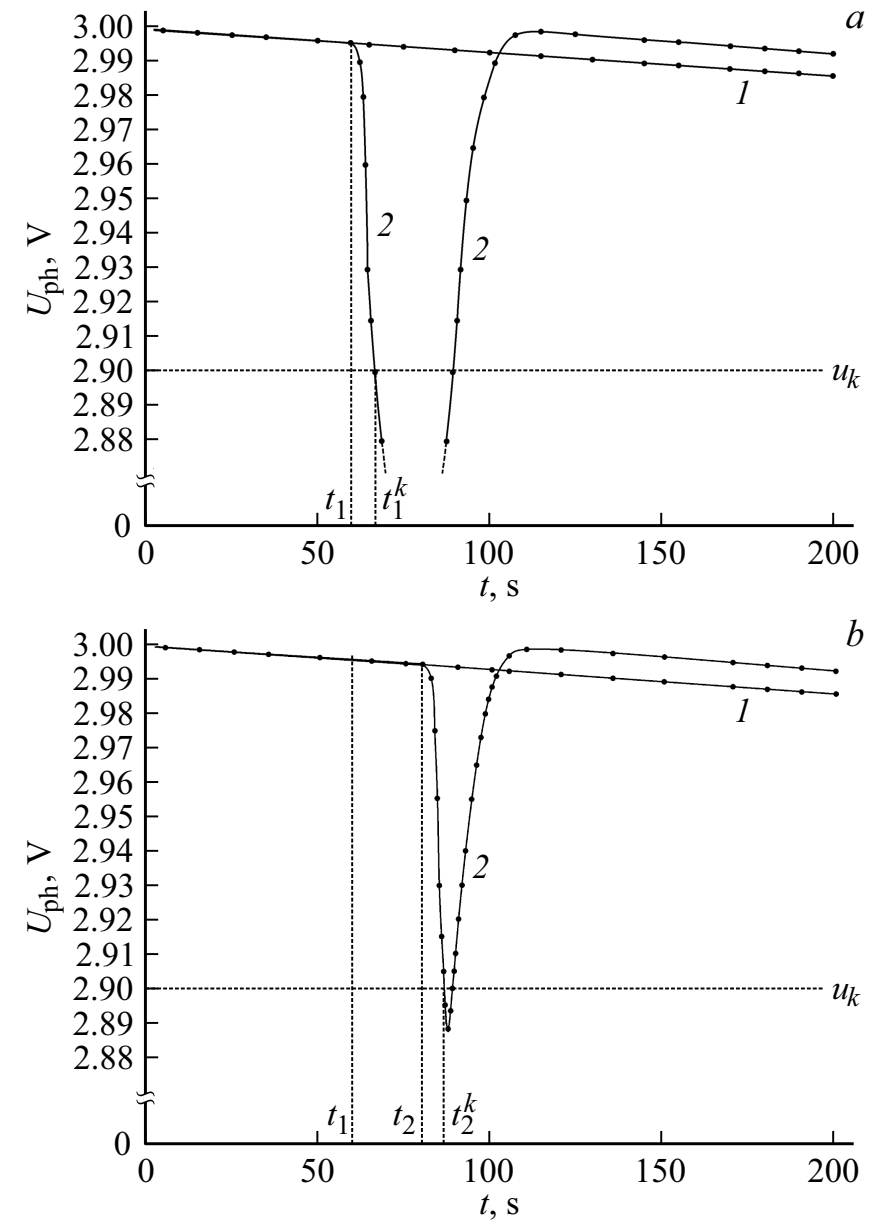

Рис. 4. Изменение напряжения $U_{\text {ph }}$ от времени $t$ на выходах фотоприемников: $a-15, b-16$ (рис. 3). График 1 соответствует случаю отсутствия в теплоносителе кислородной активности в зоне расположения катушки с оптическим волокном. График 2 - прохождению через эту зону теплоносителя с кислородной активностью (всплеск $\gamma$-квантов).

напряжение, в котором содержится информация о мощности лазерного излучения с $\lambda=1550 \mathrm{~nm}$, поступает на вход компараторов 19 и 20. На другом входе компараторов установлен уровень (значение напряжения $U_{c}$ ), при достижении которого происходит срабатывание, и на выходе компараторов формируется 1 (до этого был ноль). На рис. 4 представлены зависимости изменения напряжения $U_{\text {ph }}$ на выходах двух фотоприемников 15 и 16 от времени $t$.

Значение напряжения $U_{\mathrm{ph}}$, которое определяется мощностью лазерного излучения, регистрируемого на фотоприемниках 15 и 16, в случае воздействия на волокно только фонового радиоактивного излучения и слабых всплесков $\gamma$-квантов от потока теплоносителя уменышается медленно и за $t=250 \mathrm{~s}$ не достигает значения $U_{c}$, которое установлено на вторых входах компараторов 19 и 20 (рис. 4). Поэтому на выходе компараторов значение напряжения не изменяется и соответствует нулю. На входы микросхемы 74135 (741S35), исключа- 
ющей „ИЛИ“, которое входит в состав логического устройства 21, с выходов компараторов поступают нули. На выходе 21 формируется ноль, который поступает на устройство управления и обработки 22. В момент времени $t_{1}$ (появление большого числа $\gamma$-квантов от распада ядер ${ }^{16} \mathrm{~N}$ в зоне катушки 2 , рис. 3) значение $\alpha_{s}$ резко возрастает, значение $U_{\mathrm{ph}}$ на фотоприемнике 15 уменьшается и через некоторое время достигает $U_{c}$ на втором входе компаратора 19. Компаратор срабатывает, и на его выходе появляется высокий уровень, соответствующий логической единице, которая поступает на один из входов микросхемы, исключающей „ИЛИ“. На выходе 21 формируется сигнал, соответствующий высокому уровню (единице). Через некоторое время $\Delta t$ данный процесс происходит во втором плече схемы (катушка 3). После того как на второй вход микросхемы, исключающей „ИЛИ“, с выхода компаратора 20 поступит сигнал, соответствующий логической единице, на выходе микросхемы, исключающей „ИЛИ“, сформируется сигнал, соответствующий логическому нулю. В итоге на выходе 21 сформируется прямоугольный импульс с длительностью $\Delta t=t_{2}^{k}-t_{1}^{k}$, которая соответствует времени протекания сегмента теплоносителя длиной $L$ (рис. 3). Это меточный принцип измерения расхода жидкости или скорости потока в трубопроводах, который является самым точным по сравнению с другими [23-26].

Тогда значение расхода теплоносителя $q$ определяется с использованием следующего соотношения:

$$
q=\frac{\pi d_{p}^{2}}{4} \frac{L}{\Delta t},
$$

где $d_{p}^{2}-$ внутренний диаметр трубопровода.

После поступления в 22 прямоугольного импульса в нем формируется команда, которая запускает драйвер питания 7 , и излучение от полупроводникового лазера 8 с $\lambda=457 \mathrm{~nm}$ поступает через делитель 10 на входы мультиплексоров 12 и 13, а далее в оптическое волокно. Это излучение разрушает образовавшиеся под действием $\gamma$-излучения центры окраски и ,электронные“ соединения. Процесс релаксации становится намного быстрее образования новых центров окраски и „электронных“ соединений даже в присутствии новой кислородной активности в зоне катушки с оптическим волокном. Мощность лазерного излучения $\lambda=457 \mathrm{~nm}$, прошедшего через оптическое волокно катушек 2 и 3 , измеряется с использованием 17 и 18. Далее информация с 17 и 18 поступает в 22, где с использованием (1) определяется значение $\alpha_{s}$. При достижении определенного значения $\alpha_{s}$, которое устанавливается заранее (соответствует состоянию оптического волокна до появления мощных всплесков $\gamma$-квантов в зоне его размещения), драйвер питания 7 отключается.

Во время восстановления свойств (прозрачности оптического волокна) полупроводниковый лазер с $\lambda=1550 \mathrm{~nm}$ работает в непрерывном режиме. В определенный момент времени напряжение с фотоприемников будет больше опорного напряжения на двух других входах компараторов. Компараторы вернутся в исходное состояние (на выходе компаратора уровень напряжения будет соответствовать нулю). Это изменение на компараторах произойдет одновременно, так как процесс восстановления свойств оптического волокна в двух катушках идентичен. После этого разработанная нами система готова к новому измерению расхода $q$.

Для исследования кислородной активности теплоносителя была установлена катушка 25. Есть два варианта ее расположения. Первый, катушку 25 устанавливают перед экраном 4 (до катушки 2) и размещают между двумя экранами 27 и 28. Второй случай, катушку 25 можно разместить после экрана, который размещается за катушкой 3. Первый случай более предпочтительный, так как он позволяет получить дополнительную информацию о мощности лазерного излучения, которую можно в дальнейшем использовать для измерения расхода $q$. Для определения числа $\gamma$-квантов $N$ используют формулу (6).

На рис. 5 в качестве примера представлена зависимость изменения $N$ в зоне размещения катушки 25 от времени $t$ для различных значений $q$. В качестве теплоносителя использовался раствор $\left(\mathrm{H}_{2} \mathrm{O}+\mathrm{H}_{3} \mathrm{BO}_{3}\right)$ с нитрид плутониевым наполнением при температуре $T_{c}=960 \mathrm{~K}$

Анализ полученных на рис. 5 результатов показывает, что кислородная активность в теплоносителе распределяется во времени случайным образом. Максимальный интервал между ее всплесками в потоке теплоносителя, полученный ранее другими учеными, подтвержден. Установлено, что, регулируя $q$, можно частично управлять функцией распределения $N$ в зоне регистрации $\gamma$ квантов катушками с оптическим волокном.

Необходимо отметить, что разработанная нами волоконно-оптическая система позволяет определять число испущенных квантов в диапазоне от $10^{5}$ до $10^{14}$ в отличие от разработанных ранее приборов, которые измеряют изменение $N$ в диапазоне не более трех порядков. Этот факт существенно расширяет возможности исследования кислородной активности в потоке теплоносителя.

\section{Заключение}

Полученные результаты показали, что предложенный нами новый метод и разработанная для его реализации волоконно-оптическая система позволяют проводить исследования кислородной активности в потоке теплоносителя при различных режимах работы реактора. Данные о характере изменения кислородной активности позволяют контролировать состояние теплоносителя. Это особенно важно при проведении различных экспериментальных исследований, а также для разработки рекомендаций по улучшению конструкции действующих реакторов. 

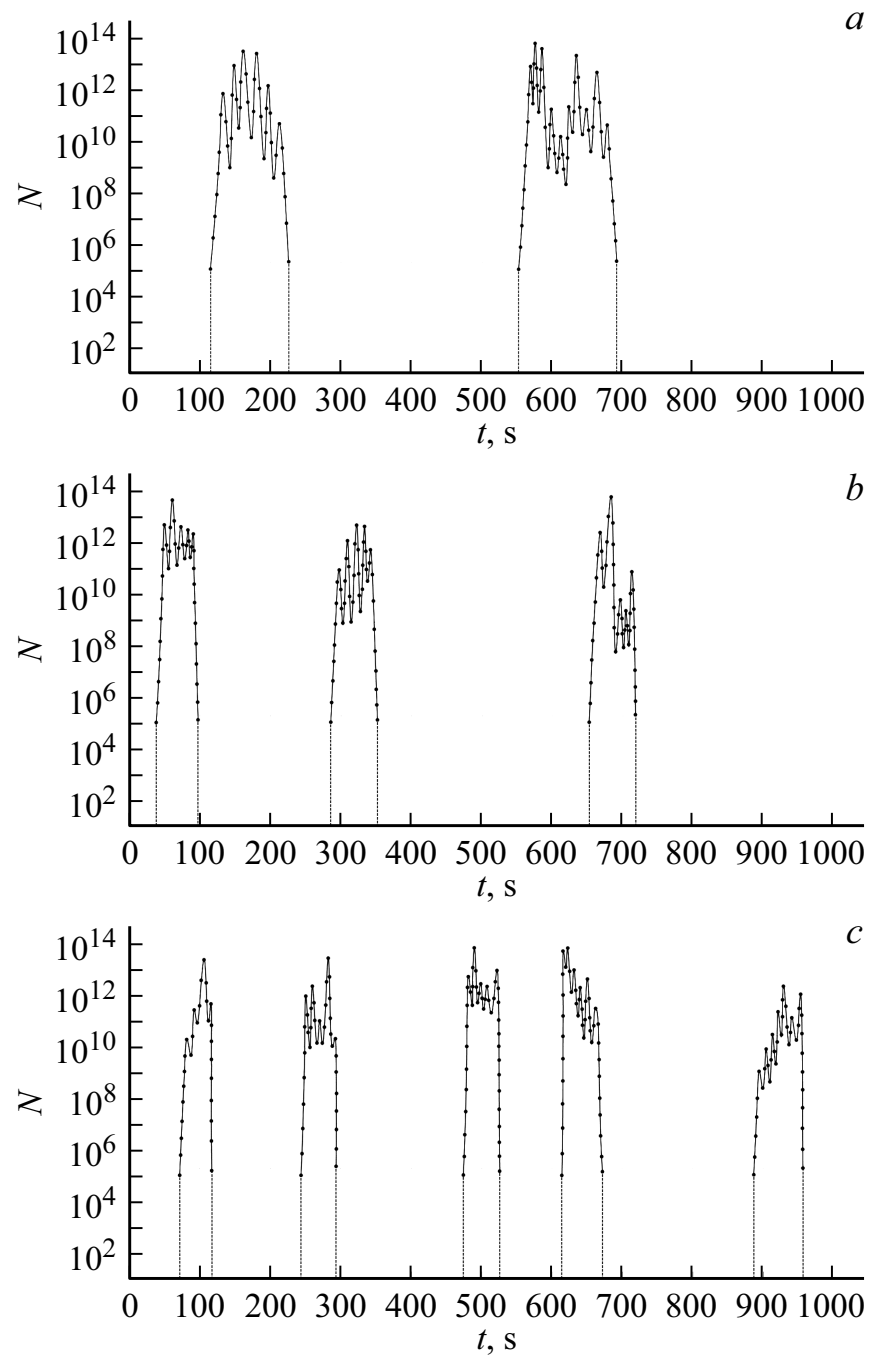

Рис. 5. Изменение числа $N$ регистрируемых $\gamma$-квантов кислородной активности от времени $t$. Графикам на рис. $a, b$ и $c$ соответствует расход теплоносителя $q\left(\right.$ в $\left.\mathrm{m}^{3} / \mathrm{s}\right): 0.169,0.339,0.678$.

Сравнение результатов измерений $q$ теплоносителя с помощью разработанной нами волоконно-оптической системы с результатами измерений его расхода электромагнитным и кариолисовым расходомерами показали высокую эффективность предложенного нами метода измерения $q$ (погрешность измерения составляет не более $1.5 \%$ ). В используемых в настоящее время промышленных расходомерах для измерения $q$ погрешность измерения составляет более 5\%. Полученный результат позволяет нам и другим ученым при проведении исследований уточнить ряд факторов, которые связаны с влиянием изменения расхода теплоносителя $q$ на число испускаемых $\gamma$-квантов $N$ в заданный промежуток времени, особенно при различных значениях $T_{c}$.

Вопрос деградации оптического волокна, которая при продолжительных исследованиях кислородной активности обязательно будет и приведет к невозможности использования данного волокна для измерений (поэтому потребуется его замена, предположительно это можно делать необходимое число раз за время эксплуатации реактора), в настоящей работе подробно не рассмотрен. Продолжительные исследования, которые необходимы для этого, еще не проводились. Это будет предметом наших дальнейших исследований.

\section{Конфликт интересов}

Авторы заявляют, что у них нет конфликта интересов.

\section{Список литературы}

[1] И.И. Семидоцкий, С.Н. Антонов, В.А. Жителев, Н.П. Котов, В.М. Махин, Б.В. Кебадзе, В.А. Шурупов. Атомная энергия, 110 (5), 262 (2011).

[2] S.V. D'yachenko, A.I. Zhernovoi. Technical Physics, 61 (12), 1835 (2016). DOI: 10.1134/S1063784216120112

[3] D.L. Griscom. Optic Materials Express, 1(3), 400 (2011). DOI: 10.1364/OME.1.000400

[4] V.V. Davydov, N.S. Myazin, A.V. Kiryukhin. Atomic Energy, 127 (5), 274 (2020). DOI: 10.1007/s10512-020-00623-5

[5] Л.В. Абрамов, А.В. Бакланов, А.М. Бахметьев. Атомная энергия, 129 (2), 105 (2020).

[6] А.П. Сорокин, Ю.А. Кузина. Атомная энергия, 128 (5), 259 (2020).

[7] S. Girard, J. Kuhnhenn, A. Gusarov, B. Brichard, M. Van Uffelen, Y. Ouerdane, A. Boukenter, C. Marcandella. IEEE Transactions on Nuclear Science, 60 (3), 2015 (2013). DOI: $10.1109 /$ TNS.2012.2235464

[8] В.И. Дудкин, В.Ю. Петрунькин, С.В. Рубинов, Л.И. Успенский. ФТТ, 26 (1), 1296 (1986).

[9] П.Ф. Кашайкин, А.Л. Томашук, М.Ю. Салганский, И.С. Азанова, М.К. Цибиногина, Т.В. Димакова, А.Н. Гурьянов, Е.М. Дианов. ЖТФ, 89 (5), 752 (2019). DOI: $10.21883 /$ JTF.2019.05.47480.123-18

[10] P.F. Kashaykin, A.L. Tomashuk, M.Y. Salgansky, A.N. Guryanov, E.M. Dianov, J. Appl. Phys., 121 (21), 213104 (2017). DOI: $10.1063 / 1.4984601$

[11] A.L. Tomashuk, M.O. Zabezhailov. J. Appl. Phys., 10(8), 083103 (2011). DOI: 10.1063/1.3561435

[12] J. Wen, G.-D. Peng, W. Luo, Z. Chen, T. Wang. Optics Express, 19 (23), 23271 (2011). DOI: 10.1364/OE.19.023271

[13] Y. Kim, S. Ju, S. Jeong, S.H. Lee, W.-T. Han. Optics Express, 24 (4), 3910 (2016). DOI: 10.1364/OE.24.003910

[14] S.V. Firstov et al. Quantum Electronics, 47 (12), 1120 (2017). DOI: 10.1070/QEL16521

[15] D.S. Dmitrieva, V.M. Pilipova, V.I. Dudkin, V.V. Davydov, V.Y. Rud. J. Phys.: Conference Series, 1697 (1), 012145 (2020). DOI: 10.1088/1742-6596/1697/1/012145

[16] D.S. Dmitrieva, V.M. Pilipova, V.Y. Rud. Lecture Notes in Computer Science (including subseries Lecture Notes in Artificial Intelligence and Lecture Notes in Bioinformatics), 12526 LNCS, 348 (2020). DOI: $10.1007 / 978-3-030-65729-1 \_30$

[17] R.V. Davydov, D.S. Dmitrieva, V.M. Pilipova, V.I. Dudkin, E.I. Andreeva. In: Proc. of 18 th IEEE International Conference Laser Optics 2020 (IEEE, 2020), p. 243. DOI: 10.1109/ICLO48556.2020.9285820 
[18] L. Dong, V.N. Bagratashvili, S.I. Tsypina, Y.S. Zavorotny, A.O. Rybaltovskii, P.V. Chernov, S.S. Alimpiev, Y.O. Simanovskii. Jap. J. Appl. Phys., 37 (51), 12 (1998).

DOI: $10.7567 / J J A P S .37 S 1.12$

[19] A.M. El-Sayed et al. Phys. Rev. B, 89 (12), 125201 (2014). DOI: $10.1103 /$ PhysRevB.89.125201

[20] D. Kovalev, J. Diener, H. Heckler, G. Polisski, N. Künzner, F. Koch. Phys. Rev. B, 61 (7), 4485 (2000). DOI: 10.1103/PhysRevB.61.4485

[21] N. Daldosso, M. Melchiorri, L. Pavesi, G. Pucker, F. Gourlilleau, S. Chausserie, Y. Ali Belarouci, X. Portier, C. Dufour. J. Lumin., 121 (2), 344 (2006). DOI: $10.1016 /$ j.jlumin.2006.08.083

[22] A.O. Rybaltovskiy, A.A. Ischenko, Y.S. Zavorotny, A.V. Garshev, S.G. Dorofeev, N.N. Kononov, N.V. Minaev, S.A. Minaeva, S.P. Sviridov, P.S. Timashev, I.I. Khodos, V.I. Yusupov, M.A. Lazov, V.Ya. Panchenko, V.N. Bagratashvili. J. Materials Science, 50 (5), 2247 (2015).

DOI: $10.1007 / \mathrm{s} 10853-014-8787-\mathrm{x}$

[23] М.Я. Марусина, Б.А. Базаров, А.А. Силаев, Н.П. Марусин, Е.Ю. Закемовская, А.Г. Гилев, А.В. Алексеев. Измерительная техника, 4, 62 (2014).

[24] Z.A. Dayev, L.N. Latyshev. Flow Measurement and Instrumentation, 56 (1), 18 (2017).

DOI: 10.1016/j.flowmeasinst.2017.07.001

[25] R. Looney, J. Priede. Flow Measurement and Instrumentation, 65 (1), 128 (2019). DOI: 10.1016/j.flowmeasinst.2018.11.019

[26] Г.Н. Ахобадзе. Измерительная техника, 5, 30 (2020). DOI: 10.32446/0368-1025it.2020-5-30-35 